\title{
Study on Advances of Medicine and Its Active Ingredient on the Hair Follicle of Different Animals
}

\author{
Guishan Zhang ${ }^{1}$ \\ ${ }^{1}$ Jining Agriculture University, Changchun, Jining, 130118
}

Keywords: Different Animals; Traditional Chinese Medicine; Medicine Extract; Hair Follicle

\begin{abstract}
An overview of several different extracts of traditional Chinese medicine and the impact of animal hair follicle belong to different species, thereby regulating mechanism of traditional Chinese medicine and its extract clear hair follicle development.
\end{abstract}

\section{Introduction}

Generated by the hair follicle, hair follicle cells cyclical growth, hair follicle growth cycle when an exception occurs, there will be hair loss, alopecia areata and other hair follicle related diseases. Hair follicles from the inside out into the hair shaft (hair stem, HS), inner root sheath (inner root sheath, IRS) and outer root sheath (outer root sheath, ORS), which are in turn made three more layers. Hair follicle cycle is divided into growth phase (anagen), catagen (catagen) and the resting phase (telogen). Hair follicle epithelial cells through a procedural degradation processes occur apoptosis hair cycle is a key step in updating alternately, thereby effectively extending the anagen, the hair follicle to inhibit apoptosis, maintaining hair follicle cells form promoting hair growth key to growth lies. Record books Boye, Eclipta have to be black and black hair effect. Modern medical research shows that Angelica can promote melanocyte proliferation, melanogenesis. Prepared Radix extract on melanocyte proliferation and migration have significant role in promoting. Astragalus extract can promote cell proliferation in mice, activation of tyrosinase activity, increased melanin content. Tribulus terrestris, mint, Polygonum, injustice silk child, independent living, also have a role in the activation of tyrosinase, may induce varying degrees of skin pigmentation; basal layer and in the lower part of the spine pigment cells increased. Hair follicles wool (cashmere) for the main economic traits for animals has become more important, the current impact of the medicine on the hair follicle and its extracts are more concentrated research mice, people and other animals, and the impact of the research and development of wool bag reported rarely.

\section{The Impact of Traditional Chinese Medicine Extract on Rat Skin and Hair Follicle}

Chinese medicine is often both nutritional and therapeutic dual role, mainly based on Chinese medicine and its role in promoting the active ingredients to the hair follicle growth. Adhirajanfound that leaf extract and hibiscus flower extract treated rats were higher than long hair, in addition, leaf extract and flower extract for 20 days after the rat hair follicle growth rate gradually higher end the ratio of the hair follicle; treatment 30 days, leaf extract treated hair follicles growing 67\%, 33\% termination rate of hair follicles; flower extract treated hair follicle growth rate of $60 \%, 40 \%$ termination rate of hair follicles. Matsuda found that $70 \%$ of the methanol extract of red ginseng 
effect on hair growth better than white ginseng extract and ginsenoside Rb1 reflect a strong stimulating vitality, and saponins.

Rg1 did not show a stimulation of hair growth vigor. Sun Yu studied the wild hawthorn, Ligustrum lucidum, Polyporus and Baiji alcohol extract mixture of mouse hair growth impact study found that the mixture of traditional Chinese medicine orally, medium and low dose group (30g/L and $10 \mathrm{~g} / \mathrm{L}$ ) and topical, the high-dose group $(40 \mathrm{~g} / \mathrm{L}$ and $80 \mathrm{~g} / \mathrm{L})$ compared with the control group of mice back skin from the pink to black ahead of time, the total time for the skin black extended from the skin turns black to hairy shortened intervals, and traditional Chinese medicine mixture oral dose group $(40 \mathrm{~g} / \mathrm{L})$ and topical high-dose group $(80 \mathrm{~g} / \mathrm{L})$ is more obvious; in addition, an external group of ginsenosides $(3 \mathrm{~g} / \mathrm{L}$ and $30 \mathrm{~g} / \mathrm{L}$ ) The first three days of the hair follicles in the growing Asia i .75\% ethanol in the growing group of sub-iv; the first 18 days, the total ginsenoside low dose group (3g/L) and 75\% ethanol group follicles in catagen, and high dose group $(30 \mathrm{~g} / \mathrm{L})$ in most of Asia is still growing vi follicles; therefore, whether topical or oral medicine mixture can promote hair follicle, hair growth and prolonging the hair growth. Rho [4] screened extracts of 45 kinds of plants, which found Asarum root extract showed the strongest vitality stimulate hair growth, a significant increase in Asarum root extract protein synthesis in cultured rat hair follicle cells tentacles. Willow The effects of Tribulus terrestris on mouse hair follicle melanocyte stimulating hormone expression, the study found that high doses of aqueous extract of Tribulus terrestris improves mouse hair follicles melanocyte-stimulating hormone level of expression, by up-regulating the hair follicle melanocytes stimulating hormone pathway to activate the expression of tyrosinase, proliferation, migration and melanin synthesis dormant hair follicles melanocytes. Wuqiao Yun found in safflower, angelica and other traditional Chinese medicine decoction mixed in vitro cultured mouse hair follicles hair growth play a catalytic role, can promote hair follicle bulb cells proliferation; mouse hair follicles single herb decoction in vitro hair growth and hair follicle bulb no significant effect on cell proliferation. Zhou found that ginseng extract concentration 0.005-0.1mg•1 ml-having pro C57BL/6J mouse hair follicle growth effect, wherein a concentration of $0.1 \mathrm{mg} \bullet 1 \mathrm{ml}$-time, best hair follicle growth . Yang Jie found that coral ginger extract treated mice skin hair follicle more complete, mice growing hair to transition from telogen, and accelerate hair growth rate, promote the synthesis of melanin. Kawano found that dense flower heath Nanti extract to promote the rapid growth of murine dermal papilla cells, and promote the vitality of the cell cycle, induction of telogen transition to initial growth, and promote hair growth of anagen. Jiang found that Polygonum extract Tetrahydroxystilbene -O- $\beta$-D- glucoside, induce cells to produce melanin melanin role. Kakali found that Eclipta extract can promote skin melanogenesis rats, the subcutaneous layer to increase the number of follicles and skin thickness effect. Park found that the amount of Polygonum extract through upregulation of Shh and $\beta$-catenin, the mice induced telogen hair follicle causing the anagen hair growth. Park found that ginseng fruit extract can promote dermal papilla cell proliferation, extend the anagen hair growth; In addition, ginseng fruit extract to improve the inhibition of the expression of apoptotic gene Bcl-2 and reducing the expression of pro-apoptotic gene bax of the amount. $\mathrm{Wu}$ found that Polygonum cuspidatum extract can significantly increase the number of hair follicles in mice callus.

\section{The Impact of Traditional Chinese Medicine and Extract of Pig Skin and Hair Follicle}

Zhangxing Hong found that Polygonum, Ligustrum lucidum and other Chinese herbal medicine processing pigs hair follicles, with the maintenance of hair follicle cells form in the growing season, 
it relieves doxorubicin-induced cell damage hair follicles, hair follicle cells have withered due to resistance to doxorubicin the role of death, so that the number of Chinese medicine treatment of hair follicle cell apoptosis was significantly lower than the control group; in addition, Shouwu, Ligustrum lucidum and other Chinese herbal medicine treatment group seven days cultured porcine follicular hair growth an average length of $2.445 \pm 0.03 \mathrm{~mm}$, higher than the control group $1.915 \pm 0.03 \mathrm{~mm}$. Zhou Naihui successfully VEGF eukaryotic expression vector PIRES2-EGFP-VEGF165 transiently transfected HaCaT cells, laser scanning confocal microscopy in cells expressing EGFP visible, but were confirmed by ELLSA supernatant VEGF was high the level of expression, and found that the supernatant can significantly promote the growth of hair follicles in vitro pig, pig delay into the hair follicle catagen.

\section{The Impact of Traditional Chinese Medicine and Other Extracts of Animal Skin Hair Follicle}

Extracts of Chinese literature and other animal hair follicle on other than human, mouse is relatively small. Liu Junfeng found that a traditional Chinese medicine capsules eliminate acne can reduce the diameter of the sebaceous glands and hair follicles in rabbits area, hair follicle infundibulum dilatation mitigation, showing a small amount of loose material within the hair follicle keratosis. Gu Jian Mei et al found that berberine oyster cream (containing berberine, Salvia, Yiyiren, Lithospermum, safflower) inhibit the expansion of the hair follicle, reducing hair follicles keratosis. Lee Hung found that different traditional Chinese medicine (Radix, Angelica, pseudo-ginseng, ginger, black sesame seeds, ginseng, astragalus) and proportion of Geese can promote hair follicle, cashmere Jilin goose main spot on the chest, abdomen and secondary back significantly increase the number of hair follicles, increasing down effect is obvious.

\section{Conclusion}

Single herb or medicine group paired hair follicle has a certain role in promoting. Chinese medicine and its active ingredient can function in the regulation of hair follicle some factors to hair follicle thus play a regulatory role, such as promoting hair follicle dermal papilla cells proliferation and development, extension of the hair follicle growth, accelerate the transition to the hair follicles from the telogen growth phase ; in addition, some inhibition of hair follicle apoptosis regulators, also play a role in promoting hair growth. However, further research needs on the herb extract on hair follicle regulation mechanism. Study of Chinese medicine and its extracts on human hair follicle is the aspect of the use of in vitro culture experiments, it is necessary to carry out further theoretical study to determine the mechanism of traditional Chinese medicine and its impact on human hair follicle extraction. About Chinese medicine and its extract in addition to people other than the mouse animal studies need to be further strengthened, particularly in wool, cashmere major economic value animals such as sheep, geese and the like.

\section{Reference:}

[1] N Adhirajan, T Ravi Kumar, N Shanmugasundaram, et al In vivo and in vitro evaluation of hair growth potential of Hibiscus rosa-sinensis Linn [J]. Journal of Ethnopharmacology, 2003,88: 235-239 .

[2] Matsuda H., Yamazaki M., Asanuma Y. Promotion of hair growth by ginseng radix on cultured mouse vibrissal hair follicles [J]. hytotherapy Research 2003,17 (7):. 797-800. 
[3] Sun Yu, Tu Ping, Wu Lingshen, etc. Study of wild hawthorn, Ligustrum lucidum, Polyporus and Baiji alcohol extract mixture of mouse hair growth effects [J]. Journal of Clinical Dermatology, 2005,34 (3): 147-150.

[4] Rho SS, Park SJ, Hwang SL, et al The hair growth promoting effect of Asiasari radix extract and its molecular regulation [J]. Journal of Dermatological Science 2005,38 (2):. 89-97.

[5] Yang Liu, Lu Jianwei, An Liang, ect. terrestris on mouse hair follicle melanocyte stimulating hormone expression [J]. Military Medical University, 2006,26 (12): 1777-1779.

[6] Wu Qiaoyun, Wu Xianjie, Lu France etc. safflower and angelica and other Chinese herbal medicine in vitro murine hair growth and hair follicle bulb cells proliferation [J]. Zhejiang University (Medical Edition), 2006,35 (4): 435-439.

[7] Zhou Min, Shen Min, Gu Yi. Other effects of ginseng extract on the C57BL / 6J mouse hair follicle growth [J]. Journal of Hospital Pharmacy, 2005,21 (6): 418-420.

[8] Yang Jie, Zheng Gang, Cao Yu. Effect in vitro Coral ginger extract on C3H / He mice hair growth cycle and hair follicles of mice tentacles [J]. Journal of Clinical Dermatology, 2003, 32 (4): 196-198.

[9] Kawano, M., Han, J., Kchouk, ME, et al Hair growth regulation by the extract of aromatic plant Erica multiflora [J]. Journal of Natural Medicine 2009,63 (3):. 335-339 .

[10] Jiang Z, Xu J, Long M, et al. 2,3,5,4'-tetrahydroxystilbene-2-O- $\beta$-D-glucoside (THSG) induces melanogenesis in B16 cells by MAP kinase activation and tyrosinase upregulation [J]. Life Science, 2009,85:. 345-350.

[11] Kakali Datta, Anu T. Singh, Ashok Mukherjee, et al Eclipta alba extract with potential for hair growth promoting activity [J]. Journal of Ethnopharmacology, 2009,124:. 450-456.

[12] Hye-Jin Park, Nannan Zhang, Dong Ki Park. Topical application of Polygonum multiflorum extract induces hair growth of resting hair follicles through upregulating Shh and $\beta$-catenin expression in C57BL / 6 mice [J]. Journal of Ethnopharmacology, 2011 135: 369-375.

[13] Soojin Park, Weon-Sun Shin, Jinnyoung Ho Fructus panax ginseng extract promotes hair regeneration in C57BL / 6 mice [J]. Journal of Ethnopharmacology, 2011,138:. 340-344.

[14] Xiao-bo Wu, Xian-qin Luo, Shu-ying Gu, et al The effects of Polygonum cuspidatum extract on wound healing in rats [J]. Journal of Ethnopharmacology, 2012,141:. 934-937.

[15] Zhang Xinghong. Effect of Chinese herbal medicine for hair growth and hair follicle pig doxorubicin-induced porcine follicular cell damage [D]. Nanjing: Nanjing Medical University, 2004.

[16] Zhou Naihui, Fan Weixin. vascular endothelial growth factor eukaryotic expression vector for expression in HaCaT cells [J]. Chinese Journal of Dermatology, 2005, 38 (7): 439-441.

[17] Liu Junfeng, Zhu Lu. Experimental Study of Acne Treatment of acne capsule rabbit ear model sebaceous glands, hair follicles and blood rheology of rats [J]. Chinese Journal of Integrative Medicine skin diseases, 2008,7 (3): 151 -54.

[18] Gu Jianmei, Jiang Xin. The study, ANCIENT berberine oyster cream on rabbit ears anti-acne keratosis experimental models Effect [J]. Chinese Journal of Modern Medicine, 2008,10 (4): 33-34.

[19] Li Changhong, Song Xiaofeng, Liang Zaiping, Other different types of traditional Chinese medicine and the increasing proportion of research Jilin goose down effect of [J]. Baicheng Normal University, 2009,23 (3): 62-65. 